\title{
The impact of cerebrospinal fluid viral analysis on empiric antibiotic use in children admitted to Tygerberg Children's hospital with suspected meningitis
}

\author{
Irma Kruger $^{a *}$, Jean Maritz ${ }^{b}$ and Heather Finlayson ${ }^{a}$ \\ ${ }^{a}$ Department of Paediatrics and Child Health, Stellenbosch University, Stellenbosch, South Africa \\ ${ }^{b}$ PathCare Reference Laboratory, Cape Town, South Africa \\ *Corresponding author, email: immie78@hotmail.co.uk
}

Check for updates

Background: Viral meningitis is the most common form of aseptic meningitis and requires minimal investigation and treatment. Polymerase chain reaction (PCR) has become the 'gold standard' for identifying viruses in cerebrospinal fluid and can provide rapid results. The objective of the study was to describe the aetiology and epidemiology of viral meningitis at Tygerberg Children's Hospital, as well as the impact of a positive cerebral spinal fluid (CSF) viral panel on the duration of empiric antibiotic treatment.

Methods: This was a retrospective folder review of all children aged between 29 days and 13 years who had a CSF specimen on which a viral analysis was performed from January 1, 2010 to December 31, 2014.

Results: A total of 288 specimens were identified from the laboratory database. Seventy-nine specimens were presented for data analysis. Thirty-seven specimens had a positive viral analysis. The median age was 11.3 months (IQR 3.7-49.16 months). The microscopy and chemistry results were similar for the two groups except for the CSF lymphocyte count, which was significantly higher in the group with a positive CSF viral analysis compared to those with a negative CSF viral analysis (median 52 vs. $12 \times 106 / \mathrm{l}, p=0.005)$. The most common identified virus was Epstein-Barr virus (EBV) ( $23 \%)$, followed by enterovirus (17\%). Children with a positive viral analysis tended to receive antibiotics for longer than those who had negative results $(p=0.223)$.

Conclusion: The addition of CSF viral analysis could be helpful in the management of children with meningitis, but at present appears to have little impact on the length of antibiotic use.

Keywords: antibiotics, cerebrospinal fluid, children, meningitis, PCR

\begin{abstract}
Background
Globally, preventable infectious diseases are a common cause of morbidity and mortality. The major causes of death, namely diarrhoea and pneumonia, are well described. ${ }^{1}$ Meningitis, however, is not as well described, but is estimated to be the cause in between $3 \%$ and $4 \%$ of all deaths in children younger than 5 years of age. ${ }^{2}$
\end{abstract}

The early diagnosis of meningitis in infants and children is essential to prevent morbidity and mortality, but making a clinical diagnosis can be difficult. ${ }^{3}$ Younger children often do not present with the classical findings of neck stiffness as seen in adults. In the absence of cerebrospinal fluid analysis a clear distinction cannot be made between viral and bacterial meningitis. ${ }^{4}$ A delay in the initiation of antimicrobial therapy increases the risk of morbidity and/or mortality. Guidelines, therefore, advise that cases of suspected meningitis in infants and children be started on empiric antibiotics as soon as possible. The antibiotic of choice is a third-generation cephalosporin (ceftriaxone or cefotaxime) given for 5-7 days. ${ }^{3}$

Viral meningitis is the most common form of aseptic meningitis and, with the exception of herpes simplex virus infection, is usually benign and requires minimal investigation and treatment. Viral meningitis is, however, historically difficult to diagnose and is also not routinely notified. ${ }^{5}$ Epidemiological data are, therefore, not routinely available. Few studies in Africa have reported identifying viruses from cerebrospinal fluid, but those that did estimated viruses to be responsible for $14-25 \%$ of all meningitis cases in Africa. ${ }^{2}$
The diagnosis of viral infections of the central nervous system has improved with the advent of new molecular technologies. ${ }^{4}$ Polymerase chain reaction (PCR) has become the 'gold standard' for detection of viruses in cerebrospinal fluid. ${ }^{6}$ Application of cerebrospinal fluid PCR has led to the identification of a viral pathogen in $80-85 \%$ of cases of aseptic meningitis. ${ }^{7}$ Data, however, are lacking on the clinical usefulness of viral PCR results.

Studies focusing on the impact of a positive molecular diagnosis on clinical management are restricted to studies identifying enteroviruses as cause of aseptic meningitis. ${ }^{7}$ These studies demonstrated that prospective use of molecular tests on admission decreases the duration of antibiotic use. ${ }^{10}$ The use of rapid enterovirus PCR reduced the duration of antibiotic use by a median of 1.5 days $(p<0.001)$ in children admitted with suspected meningitis. ${ }^{9}$

Though the benefits of rapid PCR diagnosis of enterovirus meningitis are described, it is not clear what impact the diagnosis of non-enterovirus pathogens has on the clinical management of patients.

Since 2010 the National Health Laboratory Service at Tygerberg Hospital uses the Seeplex ${ }^{\circledR}$ Meningitis ACE detection kit for the detection of viral pathogens in acute meningitis. This kit has three components, two of which detect viruses. The Seeplex ${ }^{\circledR}$ Meningitis V-1 detects six viruses: Herpes Simplex virus 1, Herpes Simplex virus 2, Varicella-zoster virus, Epstein-Barr virus, Cytomegalovirus, and Human Herpes Virus 6. The 
Seeplex ${ }^{\circledast}$ Meningitis V-2 component detects Human Enterovirus. ${ }^{11}$ The virology department also has an in-house multiplex for the detection of mumps and enterovirus. Specimens are processed for viruses when requested as part of clinical care.

The aim of this study was to describe the aetiology and epidemiology of viral meningitis in our hospital. We also aimed to see whether a positive cerebrospinal fluid (CSF) viral panel would have an effect on the length of antibiotic treatment. This study was the first meningitis study conducted after the introduction of the Seeplex ${ }^{\circledast}$ kit and the in-house multiplex for the detection of mumps and enterovirus. It was also the first study at the institution focusing solely on viral causes of meningitis and its impact on clinical care.

\section{Patients and methods}

\section{Setting}

The study was conducted at Tygerberg Children's Hospital in the City of Cape Town, Western Cape, South Africa. The hospital has direct access to the National Health Laboratory Service (NHLS) Medical Virology laboratory where cerebrospinal fluid is processed according to standard laboratory methods.

\section{Design}

All paediatric CSF specimens that had a viral analysis performed between January 1, 2010 and December 31, 2014 were identified from the National Health Laboratory Service database. A systematic query of the NHLS database was conducted to ensure completeness of acquired data. After excluding CSF specimens that did not meet the inclusion criteria mentioned below, the patient records of the remaining patients were requested from Tygerberg Hospital. A retrospective review of patient records was done.

\section{Ethics}

Ethics approval was obtained from the Health Research Ethics Committee at the University of Stellenbosch (S14/10/228).

\section{Inclusion criteria}

All children aged 29 days to 13 years of age admitted to Tygerberg Children's Hospital with suspected meningitis between January 1, 2010 and December 31, 2014 and who had a cerebrospinal fluid viral analysis performed were included in the study. Those with CSF results which were normal (lymphocytes $<6 \times 10^{6} / \mathrm{l}$ and neutrophils $<1 \times 10^{6} / \mathrm{l}$ ), traumatic (erythrocytes $>10000 \times 10^{6} / \mathrm{l}$ ), positive for bacteria or Cryptococcus on microscopy or culture, Cepheid ${ }^{\circledR}$ GeneXpert MTB/Rif or CLAT positive or processed outside of the National Health Laboratory Service at Tygerberg Hospital were excluded from the analysis. Children referred to step-down facilities for completion of treatment were also excluded.

Patients admitted to the intensive care units were excluded as these ill children usually have co-morbid factors or other infections that could influence the length of antibiotic use, irrespective of the results of a CSF viral analysis. It was also not possible to evaluate the duration of antibiotic use in children referred to step-down facilities as the researcher did not have access to clinical records in these facilities.

\section{Statistical analysis}

The data were organised in an Excel spreadsheet (Microsoft Corp, Redmond, WA, USA) for the purposes of statistical analysis. Data were analysed using STATA version 13 (StataCorp 2013.
College Station, TX, USA). A $p$-value of $<0.05$ was considered significant. Categorical variables were described using frequencies and percentages. Quantitative data were described using median and interquartile range (IQR). The relationship between HIV status and viral analysis result was described using Fisher's exact test, while the relationship between viral analysis result and duration of antibiotic use, and the relationship between HIV status and duration of antibiotic use were analysed using the Mann-Whitney U-test.

\section{Results}

Two hundred and eighty-eight culture-negative specimens that had a CSF viral analysis performed were identified. After exclusion for duplicate patient specimens, traumatic taps, normal CSF, incomplete data and remaining neonates, a total of 79 patients remained for data analysis (Figure 1). There were 36 specimens positive on viral PCR. The most common was EBV with 18 specimens, 8 were herpes viral panel positive, 9 enterovirus positive and 5 with dual infection (Table 1). After further review of the specimens, those that were positive for EpsteinBarr virus only were also excluded from the analysis. Thus 19 specimens remained positive and were included in the analysis. Forty-two specimens were negative (Table 2). The male:female ratio was $1.65: 1$. The median age was 11.3 months (IQR 3.749.16 months). Antibiotics were started in $87 \%$ of cases (53/ 61). Ceftriaxone was the most commonly used in $42 \%$ of cases (22/53). Empiric acyclovir was started in one case (negative viral panel analysis). Anti-tuberculosis treatment was started together with antibiotics in $30 \%$ of cases $(18 / 61)$ and consequently stopped again in 10 of the 18 cases; in the remainder

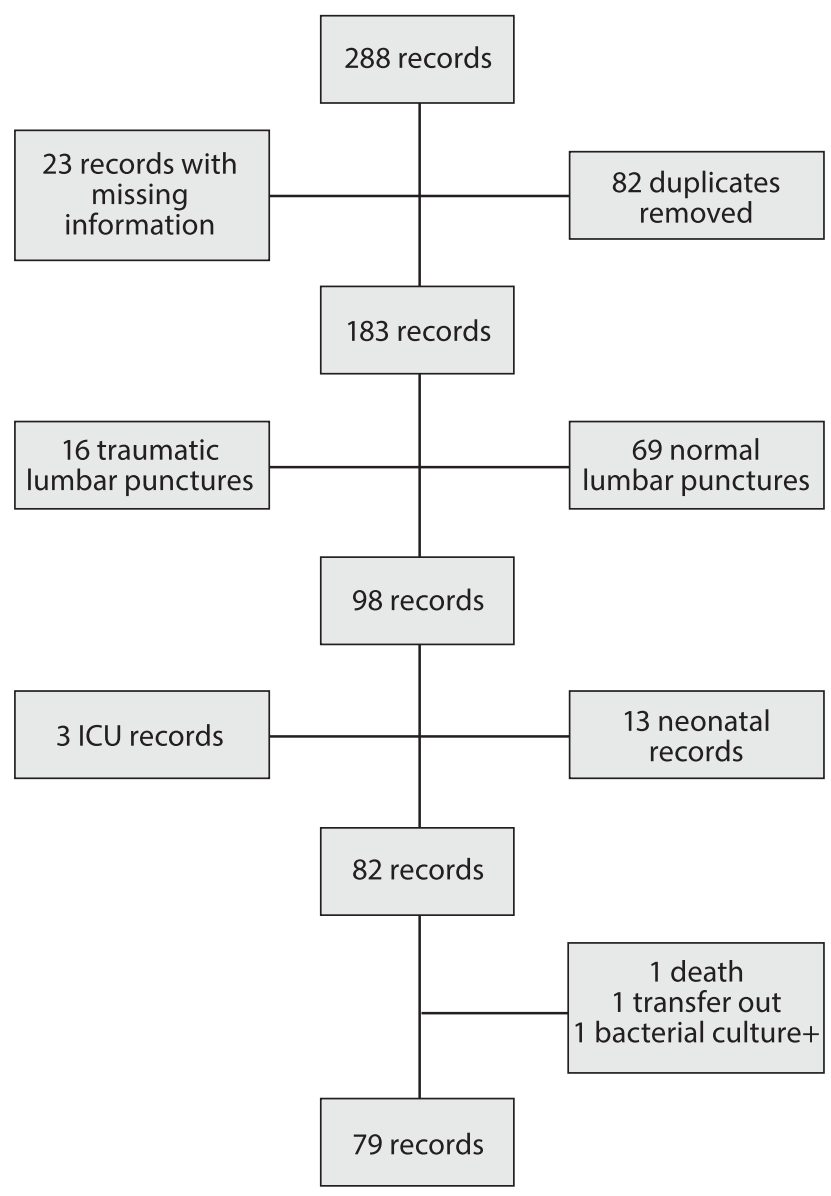

Figure 1: Flow diagram showing selection of specimens for inclusion in the study. 
Table 1: Aetiology of viral meningitis

\begin{tabular}{|l|c|c|}
\hline Isolate & Number & $\%$ of total isolates \\
\hline Negative & 42 & $53 \%$ \\
\hline Epstein-Barr virus & 18 & $24 \%$ \\
\hline Enterovirus & 9 & $11 \%$ \\
\hline Herpes simplex virus & 1 & $1 \%$ \\
\hline HHV-6 & 4 & $4 \%$ \\
\hline Dual infection & 5 & $6 \%$ \\
\hline Total & 79 & \\
\hline
\end{tabular}

Table 2: Epidemiology of viral meningitis
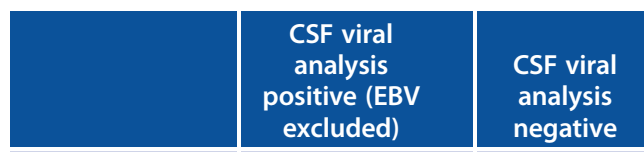

negative

excluded)

n

Male:female

ratio

Age (median)

Median PMN

count

Median

lymphocyte

count

Median protein

level

Median glucose

level

Median

duration of

antibiotic use

\begin{tabular}{|c|c|c|}
\hline 19 & 42 & 61 \\
\hline $2.8: 1$ & $1.33: 1$ & $1.65: 1$ \\
\hline 8.8 months & 13.8 months & $\begin{array}{c}11.3 \\
\text { months }\end{array}$ \\
\hline $3 \times 10^{6} / /$ & $1 \times 10^{6} / /$ & $2 \times 10^{6} / \mathrm{l}$ \\
\hline $52 \times 10^{6} / \mathrm{l}$ & $12 \times 10^{6} / \mathrm{l}$ & $20 \times 10^{6} / \mathrm{l}$ \\
\hline $0.43 \mathrm{~g} / \mathrm{l}$ & $0.37 \mathrm{~g} / \mathrm{l}$ & $0.41 \mathrm{~g} / \mathrm{l}$ \\
\hline $3.4 \mathrm{mmol} / \mathrm{l}$ & $3.35 \mathrm{mmol} / \mathrm{l}$ & $3.4 \mathrm{mmol} / \mathrm{l}$ \\
\hline 5 days & 2.5 days & 4 days \\
\hline
\end{tabular}

of cases the anti-tuberculosis treatment was continued. A CSF cell count and chemistry was performed in all cases. The median polymorph nuclear (PMN) cell count was $2 \times 10^{6} / \mathrm{I}$ (IQR: $\left.0-19 \times 10^{6} / \mathrm{l}\right)$ and the median (SF lymphocyte count was $20 \times$ $10^{6} / \mathrm{I}$ (IQR: $6-69 \times 10^{6} / \mathrm{l}$ ). The median CSF protein level was $0.41 \mathrm{~g} / \mathrm{l}$ (IQR $0.31-0.65 \mathrm{~g} / \mathrm{l})$. The median CSF glucose was $3.4 \mathrm{mmol} / \mathrm{I}$ (IQR $2.9-4 \mathrm{mmol} / \mathrm{l})$. The median duration of antibiotic use was 4 days (IQR: $1-5$ days).

There was a trend that children with a positive CSF viral analysis were younger than the children with a negative CSF viral analysis (median 8.8 months vs. 13.8 months). This was, however, not significant $(p=0.60)$. The microscopy and chemistry results were similar for the two groups except for the CSF lymphocyte count, which was significantly higher in the group with a positive CSF viral analysis compared with those with a negative CSF viral analysis (median 52 vs. $12 \times 10^{6} / \mathrm{l}, p=0.005$ ) (Figure 2). The glucose for both groups fell within the normal range (median value 3.4 vs. $3.35 \mathrm{mmol} / \mathrm{l}, p=0.76$ ), the median protein was normal in the negative CSF group and only slightly raised in the positive group ( $0.37 \mathrm{~g} / \mathrm{l}$ vs. $0.43 \mathrm{~g} / \mathrm{l}, p=0.45)$.

A larger proportion of children with a negative CSF viral panel analysis ( $16 \%$ vs. $5 \%$ in viral panel positive patients) were never started on antibiotics. Children with a positive viral analysis received antibiotics for longer than those that had a negative result (median 5 days; IQR 2-5 days vs 2.5 days; IQR $1-5$ days); however, this was not significant $(p=0.223)$.

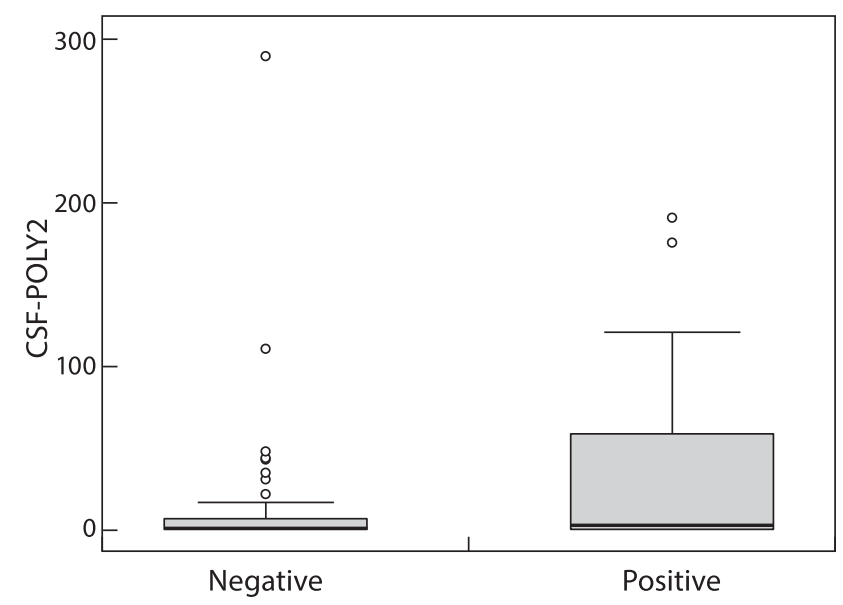

Figure 2: Box plot of CSF lymphocyte cell count distribution for positive and negative viral panels.

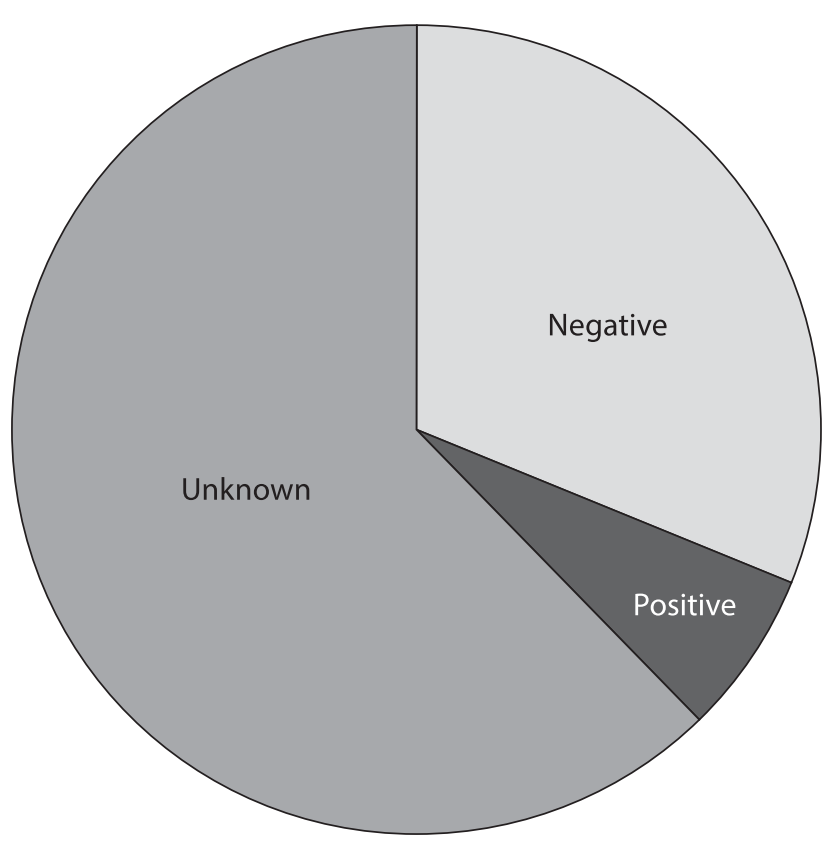

Figure 3: HIV status during admission.

The HIV status was unknown in $61 \%$ of cases (37/61), negative in $31 \%$ of cases $(19 / 61)$ and positive in $7 \%$ of cases $(4 / 61)$ (Figure 3$)$. Of those who were HIV infected, 71\% (5/7) were already receiving antiretroviral therapy. There was no relationship between HIV status and viral panel analysis result $(p=0.886)$.

\section{Discussion}

This study presents the information on viral meningitis in the paediatric population at Tygerberg Children's Hospital, Western Cape, South Africa for a five-year period from January 1, 2010 to December 31, 2014. This coincides with the period when the Seeplex ${ }^{\circledR}$ kit for CSF viral analysis became available at the institution.

Enterovirus is well known as the most common cause of viral meningitis in childhood and is best described in the literature. ${ }^{6}$ A study in Cape Town, South Africa isolated viruses in 3406 of the 11360 specimens; $91 \%$ yielded enteroviruses..$^{5}$ This was also reflected in our study. A large proportion of specimens in our study yielded EBV as an isolate. EBV genetic material can, 
however, be carried in lymphocytes after a primary infection. When the lymphocytes cross the blood-brain barrier, this genetic material will be present in the lymphocytes in the CSF. This EBV genetic material can, therefore, be picked up by the viral panel assay without there being active infection. ${ }^{12}$ EBV was, therefore, excluded from the analysis. Although the same can be true for HHV-6, it was felt that HHV- 6 can be associated with symptoms of meningitis as well as febrile seizures in infants (a common reason for lumbar puncture in the emergency care setting) and was, therefore, included in the analysis. Viral PCR results should, therefore, always be interpreted in conjunction with the clinical history as viral DNA may easily be picked up by PCR in the presence of lymphocytes.

CSF parameters in cases with a positive CSF viral analysis were similar to those with a negative viral analysis, except for lymphocyte count, which was significantly higher in positive cases ( $p=$ 0.005 ). This is to be expected as viral meningitis typically has a CSF with raised lymphocytes but normal protein and glucose values.

Our study showed a male predominance among those with a positive CSF viral panel analysis. This is in keeping with the findings of an earlier study in Cape Town. ${ }^{5}$ Even though the study excluded neonates from the analysis, the median age among those with a positive CSF viral panel analysis remains relatively low (8.8 months). This most likely reflects the young population which is seen in our casualty and short-stay ward. ${ }^{13}$

Some $61 \%(37 / 61)$ of cases with meningitis did not have an HIV status documented, thus the number of HIV infected cases was small and it was, therefore, difficult to draw inferences with regard to the epidemiology of viral meningitis in HIV infected children.

A large proportion of children in this study $(18 / 61 ; 30 \%)$ were started on concomitant TB treatment. Even though the anti-tuberculous treatment was stopped in some cases, $40 \%$ of cases were discharged with anti-tuberculous treatment and thus the diagnosis of tuberculous meningitis. This reflects the high burden of tuberculosis in the Western Cape, with TBM being the most common form of bacterial meningitis in the area. ${ }^{14}$

The literature suggests that a positive viral analysis of the CSF leads to shorter duration of antibiotic use (2.3 days shorter). ${ }^{6}$ We, however found the opposite to this, where children with a positive viral panel analysis had a median duration of antibiotic use of 5 days, compared with those with a negative viral panel analysis receiving a median duration of 2.5 days of antibiotics. This, however, was not a statistically significant finding ( $p=$ 0.223). Reasons for this longer duration may include longer turnaround times as at the time of the study, that PCRs were not processed as a 24 hour service, delayed identification of the result by clinicians as results are documented on an online service and are not phoned out, and that the initial rollout of the service was limited to Mondays, Wednesdays and Fridays, thus earlier specimens had a longer turnaround time. We were also unable to ascertain the clinical reason for stopping antibiotics as this was poorly documented in the patient folders, thus the reason for continuing or stopping antibiotics may have been unrelated to the PCR result.

\section{Study limitations}

There are a number of limitations to this study. This includes a small sample size. This is a retrospective folder review and the data quality was dependent on the availability, accessibility and quality of data kept. The study utilised consecutive sampling, including all children that meet the inclusion criteria. This could have introduced bias into the study, but there is no reason to believe that children are not admitted in a random way. The study was conducted at a single centre. Since Tygerberg Children's Hospital is a large referral centre, children with mild or moderate disease might be underrepresented in this study as they receive treatment at their local level 1 or level 2 health facilities.

\section{Recommendations}

Ongoing viral meningitis surveillance is necessary to present a clear picture on viral meningitis in childhood. A prospective study should be conducted to include clinical data, as well as data on length of stay and potential hospital cost saved due to the addition of CSF viral analysis to clinical care. Such a study design could also include the neonatal age group.

\section{Conclusion}

The addition of CSF viral analysis may be helpful in the management of children with meningitis, but at present appears to have little impact on the use of empiric antibiotics in Tygerberg Children's Hospital.

Disclosure statement - No potential conflict of interest was reported by the authors.

\section{References}

1. Liu L, Johnson $H$, Cousens $S$, et al. Global, regional, and national causes of child mortality: an updated systematic analysis for 2010 with time trend since 2000. Lancet. 2012;379(9832): 2151-2161. 10. 1016/S0140-6736(12)60560-1

2. Johnson $\mathrm{H}$, Liu L, Fischer-Walker $\mathrm{C}$, et al. Estimating the distribution of cause of death among children age 1-59 months in high-mortality countries with incomplete death certification. Int J Epidemiol. 2010;39(4): 1103-1114. 10.1093/ije/dyq074

3. Boyles T, Maford C, Bateman K, et al. Guidelines for the management of acute meningitis in children and adults in South Africa. South African Journal of Infectious Diseases 2013;28(1): 5-15.

4. DeBiasi R, Tyler K. Molecular methods for the diagnosis of viral encephalitis. Clin Microbiol Rev. 2004;17(4): 903-925. 10.1128/CMR.17.4. 903-925.2004

5. McIntyre JP, Keen GA. Laboratory surveillance of viral meningitis by examination of cerebrospinal fluid in Cape Town, 1981-9. Epidemiol Infect. 1993;111(02): 357-371. 10.1017/S095026880005706X

6. Archimbaud C, Chambon M, Bailly JL, et al. Impact off rapid enterovirus molecular diagnosis on the management of infants, children and adults with aseptic meningitis. J Med Virol. 2009;81(1): 42-48. 10.1002/jmv.21330

7. Hosoya M, Honzumi K, Sato $M$, et al. Application of $P C R$ for various neurotropic viruses on the diagnosis of viral meningitis. J Clin Virol. 1998;11(2): 117-124. 10.1016/S1386-6532(98)00048-8

8. Bhaskaran A, Racsa L, Southern P, et al. Interpretation of positive molecular tests of common viruses in the cerebrospinal fluid. Diagn Microbiol Infect Dis. 2013;77(3): 236-240. 10.1016/j.diagmicrobio. 2013.07.017

9. Ramers C, Billman G, Hartin M, et al. Impact of a diagnostic cerebrospinal fluid enterovirus polymerase chain reaction test on patient management. JAMA. 2000;283(20): 2680-2685. 10.1001/jama.283.20.2680

10. Archimbaud C, Ouchchane L, Mirand A, et al. Improvement of the management of infants, children and adults with a molecular diagnosis of enterovirus meningitis during two observational study periods. Public Library of Science ONE 2013;8(7): 1-8.

11. Shin S, Kwon K, Park J. Evaluation of the seeplex meningitis ACE detection kit for detection of 12 common bacterial and viral pathogens of acute meningitis. Annals of Laboratory Medicine. 2012;32 (1): 44-49. 10.3343/alm.2012.32.1.44

12. Kleinschmidt-DeMasters BK, DeBiasi RL, Tyler KL. Polymerase chain reaction as a diagnostic adjunct in herpes virus infections of the 
nervous system. Brain Pathol. 2001;11(4): 452-464. 10.1111/j.17503639.2001.tb00414.x

13. Finlayson $H$, Smit $L$, Esterhuizen TM, et al. The impact of a change in referral pathway on a paediatric short-stay ward in Cape Town, South Africa. South African Journal of Child Health 2016;10(2): 134-138. 10. 7196/SAJCH.2016.v10i2.1054
14. Wolzak N, Cooke M, Orth $\mathrm{H}$, et al. The changing profile of paediatric meningitis at a referral centre in Cape Town. J Trop Pediatr. 2012;58 (6): 491-495. 10.1093/tropej/fms031

Received: 24-11-2017 Accepted: 24-04-2018 